N evada

DOE/NV--550

Environmental

Restoration

Project

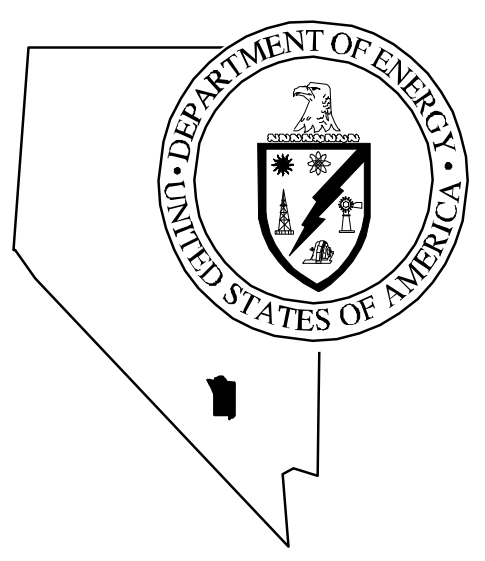

Housekeeping Closure Report for

Corrective Action Unit 212:

Area $23 \mathrm{~W}$ arehouses $E \& R$ Chemical

Storage, $\mathrm{N}$ evada Test $\mathrm{S}$ ite, $\mathrm{N}$ evada

Controlled Copy $\mathrm{N}$ 0.:

Revision N 0.: 0

May 1999

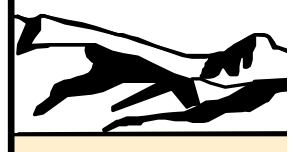

Environm ental Restoration

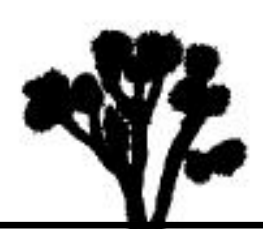

Division 
Available to the public from -

\author{
U.S. Department of Commerce \\ National Technical Information Service \\ 5285 Port Royal Road \\ Springfield, VA 22161 \\ (703) 487-4650
}

Available electronically at http://www.doe.gov/bridge. Available to U.S. Department of Energy and its contractors in paper from -

U.S. Department of Energy

Office of Scientific and Technical Information

P.O. Box 62

Oak Ridge, TN 37831-0062

(423) 576-8401 


\section{HOUSEKEEPING CLOSURE REPORT FOR CORRECTIVE ACTION UNIT 212: AREA 23 WAREHOUSES E \& R CHEMICAL STORAGE, NEVADA TEST SITE, NEVADA}

DOE Nevada Operations Office

Las Vegas, Nevada

Controlled Copy No.:

Revision No.: 0

May 1999 


\section{HOUSEKEEPING CLOSURE REPORT FOR}

CORRECTIVE ACTION UNIT 212:

\section{AREA 23 WAREHOUSES E \& R CHEMICAL STORAGE,} NEVADA TEST SITE, NEVADA

Approved by:

Signature Approved

$5 / 7 / 99$

Date:

Janet Appenzeller-Wing, Project Manager

Industrial Sites Project

Signature Approved

$5 / 7 / 99$

Approved by:

Date:

Runore C. Wycoff, Division Director

Environmental Restoration Division 


\section{FFACO CORRECTIVE ACTION UNIT Closure Report Summary}

FACILITY NAME: NeVAdA TeSt Site, AREAS 22 AND 23

CORRECTIVE ACTION UNIT NUMBER: 212

SITES: HOUSEKEEPING, INCLUDING: DRUMS, BATTERIES, SCRAP METAL, AND OTHER MATERIALS

CORRECTIVE ACTION SITE Numbers: 22-02-01

22-19-02

22-24-01

23-18-01

23-18-02

23-22-01 


\section{FFACO CORRECTIVE ACTION Site HouseKeEPING Closure VERIFICATION FoRM}

Closure Verification Date: 12 February 1998

CAS Number: 22-02-01

General Location: Desert Rock Airstrip

Latitude: $36.6276656 \mathrm{E}$

Longitude: $-116.0276166 \mathrm{E}$

\author{
CAU Number: 212 \\ Elevation: $976 \mathrm{~m}$ \\ Northing: 4053807 \\ Easting: 586942
}

Coordinate/Elevation Data Obtained from NORTH AMERICAN DATUM, 1927.

Site Access Route: Travel west on Camp Desert Rock Road from Mercury Highway. Drive to the north end of the airstrip and turn left (southwest) on a gravel road. Travel approximately 500 feet to a small building. The Underground Storage Tank (UST) was located between the building and the former propane tank guard poles.

\begin{tabular}{|l|l|}
\hline \multicolumn{1}{|c|}{ Waste Item(s) Originally at Site } & \multicolumn{1}{c|}{ Apparent Waste Type* $^{\text {A }}$} \\
\hline UST & Other \\
\hline
\end{tabular}

* Ordinary, Scrap Metal, Asbestos, PCB, Salvageable, Hazardous, Radioactive, Mixed, Unknown, Other

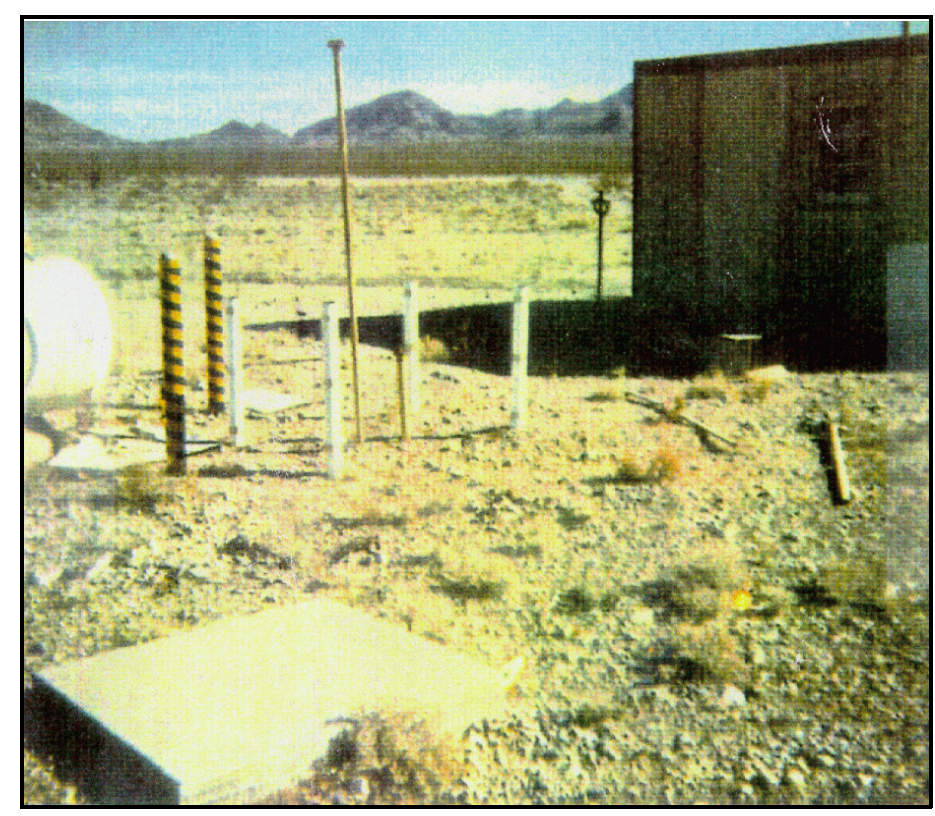

CAS Prior to Cleanup

Photograph date: 01/24/1991

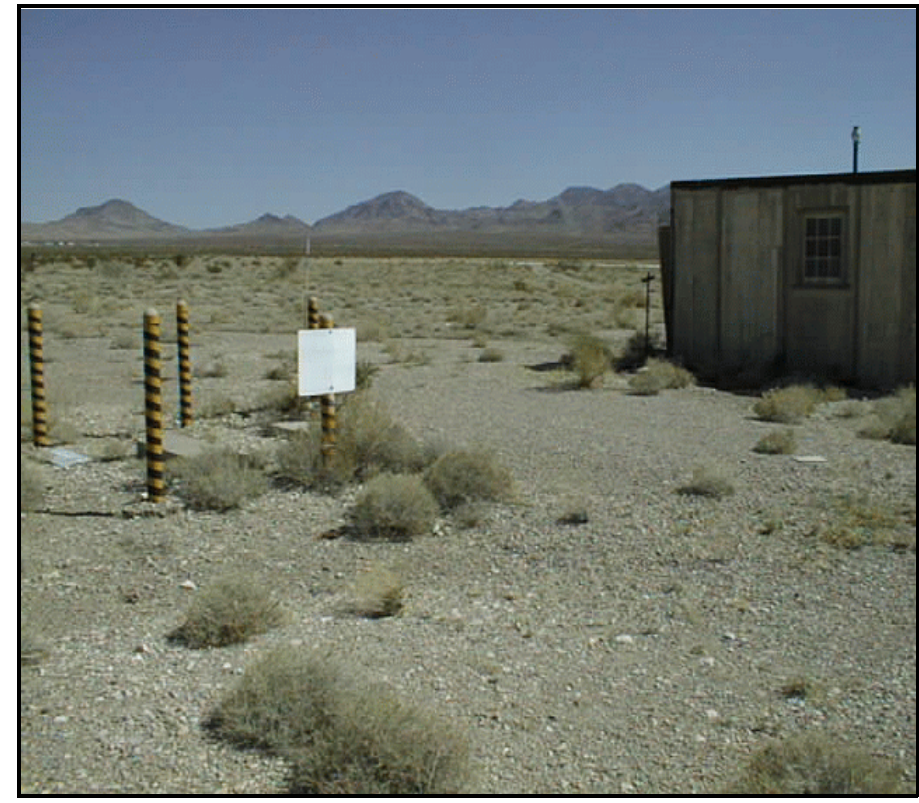

CAS After Cleanup

Photograph date: 03/04/1999

Current Site Description/Observations: The UST contents

were evacuated on 09/30/1991 and the UST was removed on 11/01/1991. The tank excavation has been back filled and there is no observed debris present at the site. Nevada Division of Environmental Protection UST Division was notified of the tank closure with EPA Form 7530-1. No Further Action Required at Corrective Action Site

$\underline{\text { Brad Jackson (Industrial Sites Project Manager) }}$ 


\section{FFACO CORRECTIVE ACTION Site Housekeeping Closure Verification Form}

Closure Verification Date：12 February 1998

CAS Number: 22-19-02

General Location: Camp Desert Rock

CAU Number: 212

Latitude: 36.6202774E

Longitude: $-116.0182373 \mathrm{E}$

Elevation: $969 \mathrm{~m}$

Northing: 4052996

Easting: 587789

Coordinate/Elevation Data Obtained from NORTH AMERICAN DATUM, 1927.

Site Access Route: Travel west on the Desert Rock Access Road from Mercury Highway. Proceed on the Desert Rock Access

Road to the weather station on the left (south). The site is located approximately 650 yards south of the weather station.

\begin{tabular}{|l|l|}
\hline \multicolumn{1}{|c|}{ Waste Item(s) Originally at Site } & \multicolumn{1}{|c|}{ Apparent Waste Type* $^{\text {Aptap }}$} \\
\hline Scrap metal and military vehicle parts & Scrap metal \\
\hline
\end{tabular}

* Ordinary, Scrap Metal, Asbestos, PCB, Salvageable, Hazardous, Radioactive, Mixed, Unknown, Other

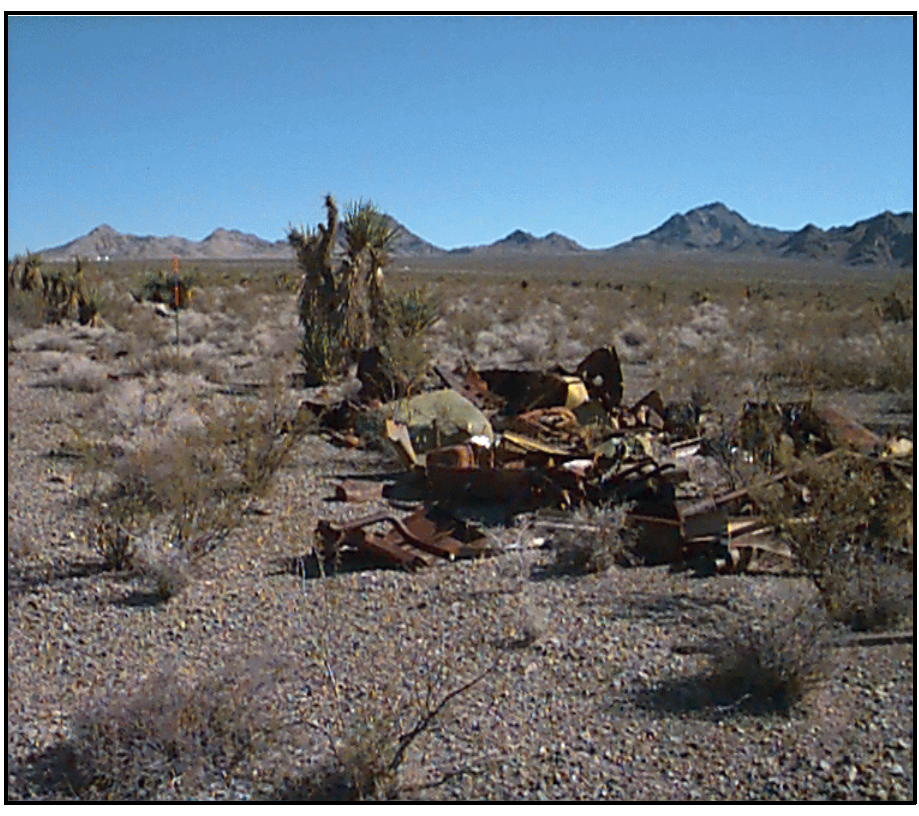

CAS Prior to Cleanup

Photograph date: 03/04/1997

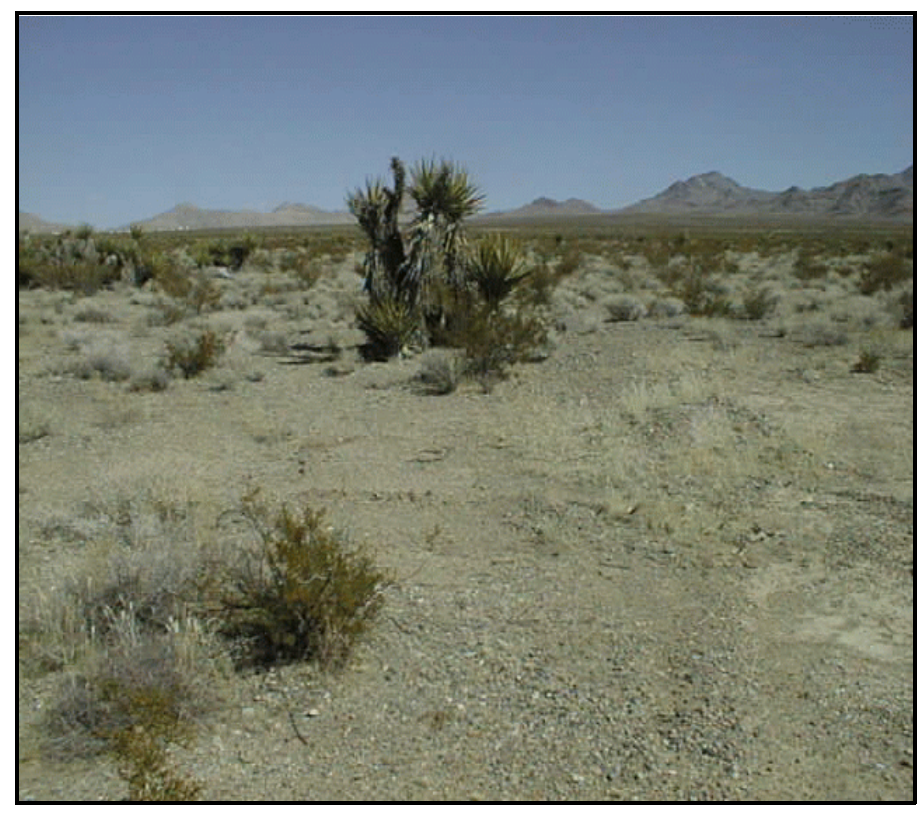

CAS After Cleanup

Photograph date: 03/04/1999

Current Site Description/Observations: The debris was removed from the site and documented in the Housekeeping Closure

Verification Form for CAU 353, CAS 22-99-03 dated 09/05/1997. There is no evidence of remaining debris.

\section{T No Further Action Required at Corrective Action Site}

Brad Jackson (Industrial Sites Project Manager) 


\section{FFACO CORRECTIVE ACTION Site Housekeeping Closure Verification Form}

Closure Verification Date: 23 February 1998

CAS Number: 22-24-01

General Location: Area 22

Latitude: 36.609366667E

Longitude: $-116.052610000 \mathrm{E}$

\author{
CAU Number: 212 \\ Elevation: $990 \mathrm{~m}$ \\ Northing: 4051755 \\ Easting: 584727
}

Coordinate/Elevation Data Obtained from NORTH AMERICAN DATUM, 1927.

Site Access Route: Proceed 4.4 miles southwest on Jackass Flats Road, from Mercury. Turn left (south) on the paved road connecting to I-95 and proceed 1.6 miles to the site located 30 feet to the right (west) of the road.

\begin{tabular}{|l|l|}
\hline \multicolumn{1}{|c|}{ Waste Item(s) Originally at Site } & \multicolumn{1}{c|}{ Apparent Waste Type* } \\
\hline One large lead acid battery, intact & Hazardous \\
\hline
\end{tabular}

* Ordinary, Scrap Metal, Asbestos, PCB, Salvageable, Hazardous, Radioactive, Mixed, Unknown, Other

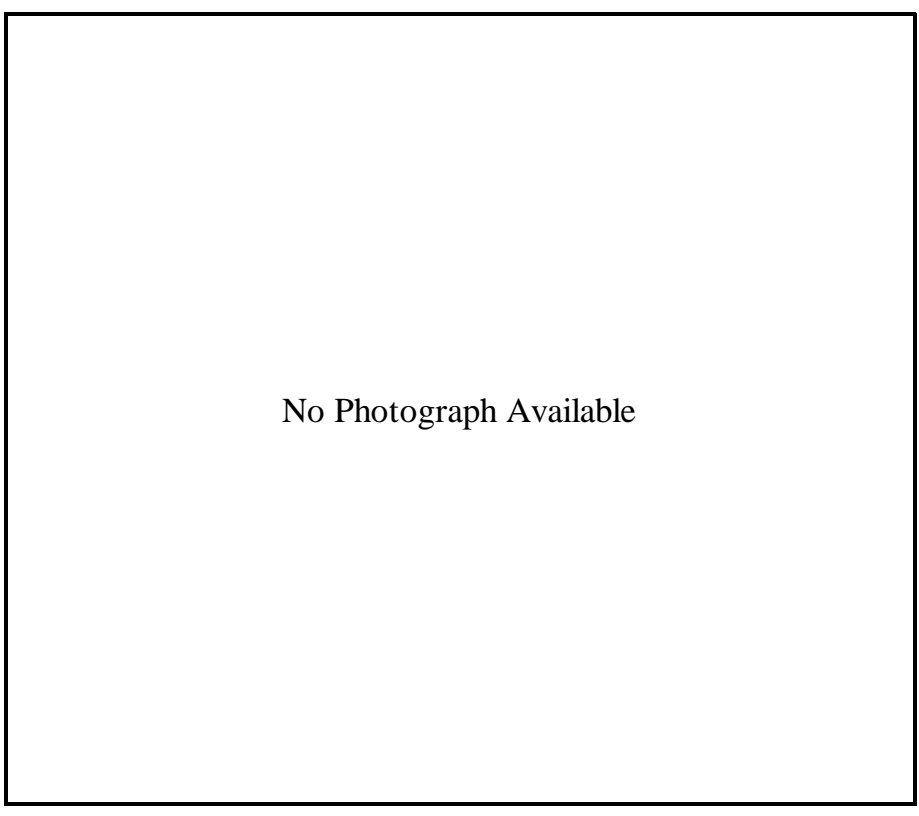

CAS Prior to Cleanup

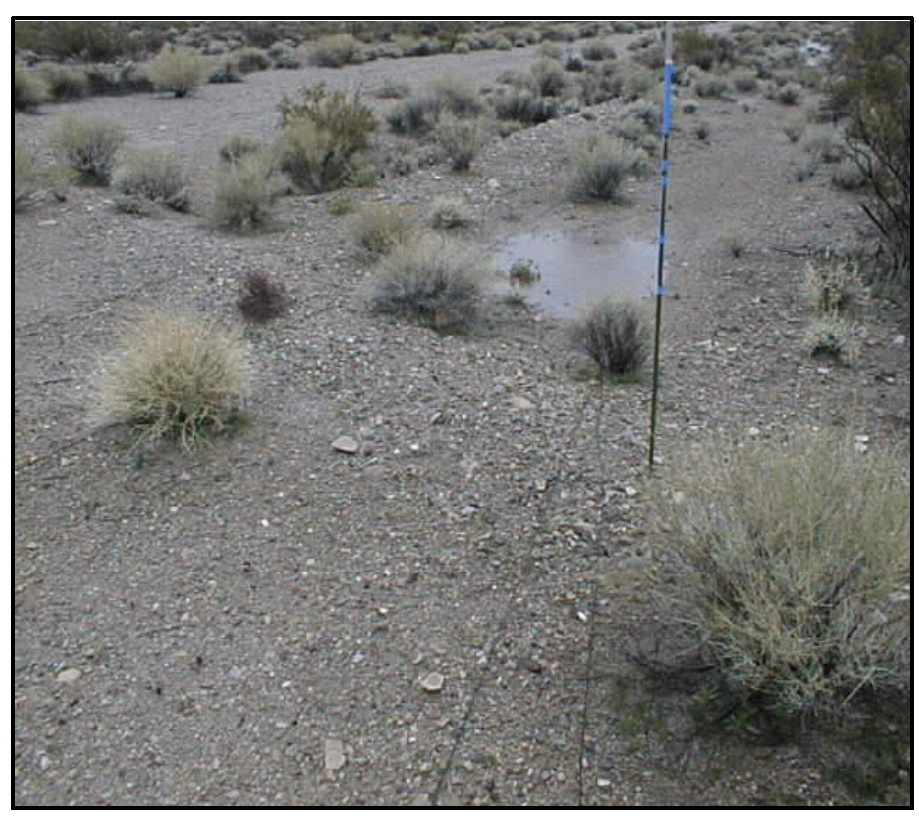

CAS After Cleanup

Photograph date: 02/23/1998

Current Site Description/Observations: The intact battery was removed. No apparent soil staining was noted during site photography on 02/23/1998.

$\mathbf{T}$ No Further Action Required at Corrective Action Site

Brad Jackson (Industrial Sites Project Manager)

Corrective Action Coordinator/Designee

(Signature)

Date 


\section{FFACO CORRECTIVE ACTION SITE HousekeEPing Closure Verification Form}

Closure Verification Date: 19 February 1998

CAS Number: 23-18-01

CAU Number: 212

General Location: Building 160, Mercury

Elevation: $1,130 \mathrm{~m}$

Latitude: $36.66400144 \mathrm{E}$

Northing: 4057868

Longitude: $-115.9953452 \mathrm{E}$

Easting: 589786

Coordinate/Elevation Data Obtained from NORTH AMERICAN DATUM, 1927.

Site Access Route: Travel east on Warehouse Road from Mercury Highway. Building 160 (Warehouse I) is on the right (south) side of the road. The site is located in the southern end of the Building 160 yard, in row 2, yard 1, section C.

\begin{tabular}{|l|l|}
\hline \multicolumn{1}{|c|}{ Waste Item(s) Originally at Site } & \multicolumn{1}{|c|}{ Apparent Waste Type* } \\
\hline Flammable solvents & Hazardous \\
\hline
\end{tabular}

* Ordinary, Scrap Metal, Asbestos, PCB, Salvageable, Hazardous, Radioactive, Mixed, Unknown, Other

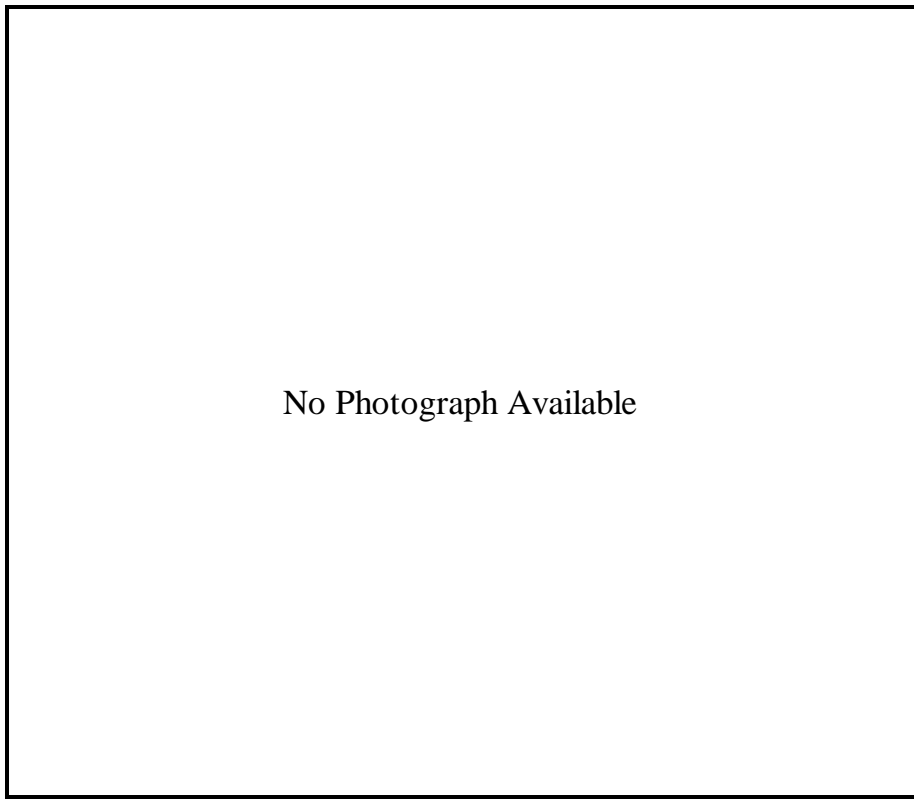

CAS Prior to Cleanup

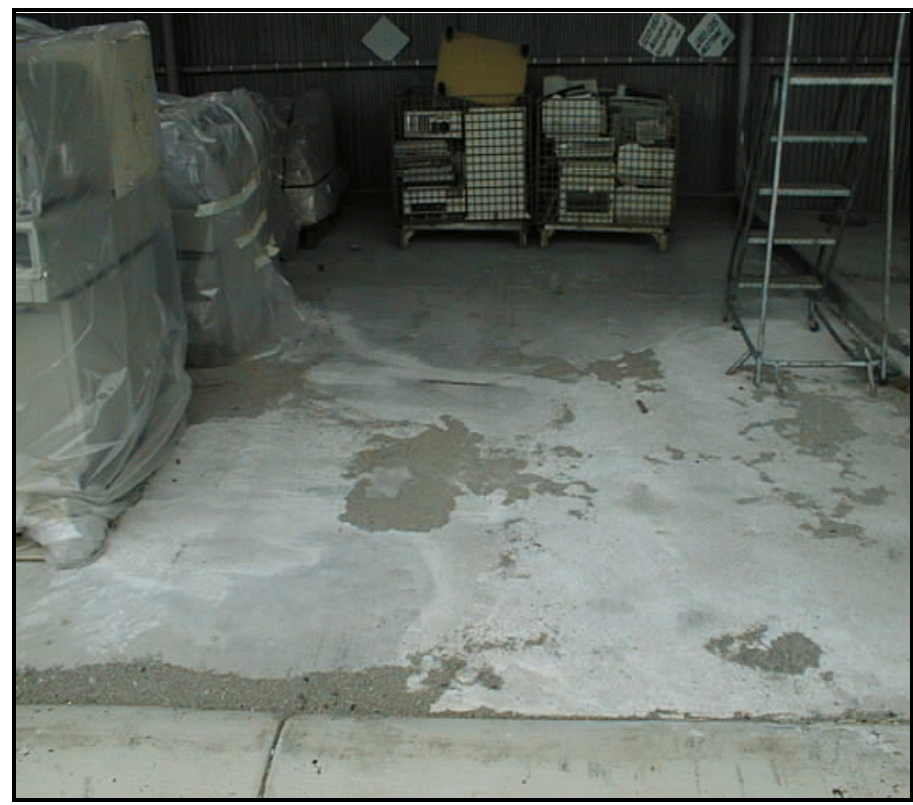

CAS After Cleanup

Photograph date: 02/19/1998

Current Site Description/Observations: The flammable solvents were "issued products" for future use and were not waste. The improperly stored flammable solvents were removed and properly stored in a covered area by J. McDow (REECo). These compliance actions were approved by DOE/NV on 10/17/1989.

\section{$\underline{T}$ No Further Action Required at Corrective Action Site}




\section{FFACO CORRECTIVE ACTION SITE HousekeEPING Closure Verification Form}

Closure Verification Date: 05 February 1998

CAS Number: 23-18-02

CAU Number: 212

General Location: Warehouse R, Mercury

Elevation: $1,132 \mathrm{~m}$

Latitude: $36.66463988 \mathrm{E}$

Northing: 4057937

Longitude: $-115.9975286 \mathrm{E}$

Easting: 589590

Coordinate/Elevation Data Obtained from NORTH AMERICAN DATUM, 1927.

Site Access Route: Proceed west on Warehouse Road from Mercury Highway. Warehouse R and E are on the right (north) side of the road.

\begin{tabular}{|l|l|}
\hline \multicolumn{1}{|c|}{ Waste Item(s) Originally at Site } & \multicolumn{1}{|c|}{ Apparent Waste Type* } \\
\hline Various acids and bases & Hazardous \\
\hline
\end{tabular}

* Ordinary, Scrap Metal, Asbestos, PCB, Salvageable, Hazardous, Radioactive, Mixed, Unknown, Other

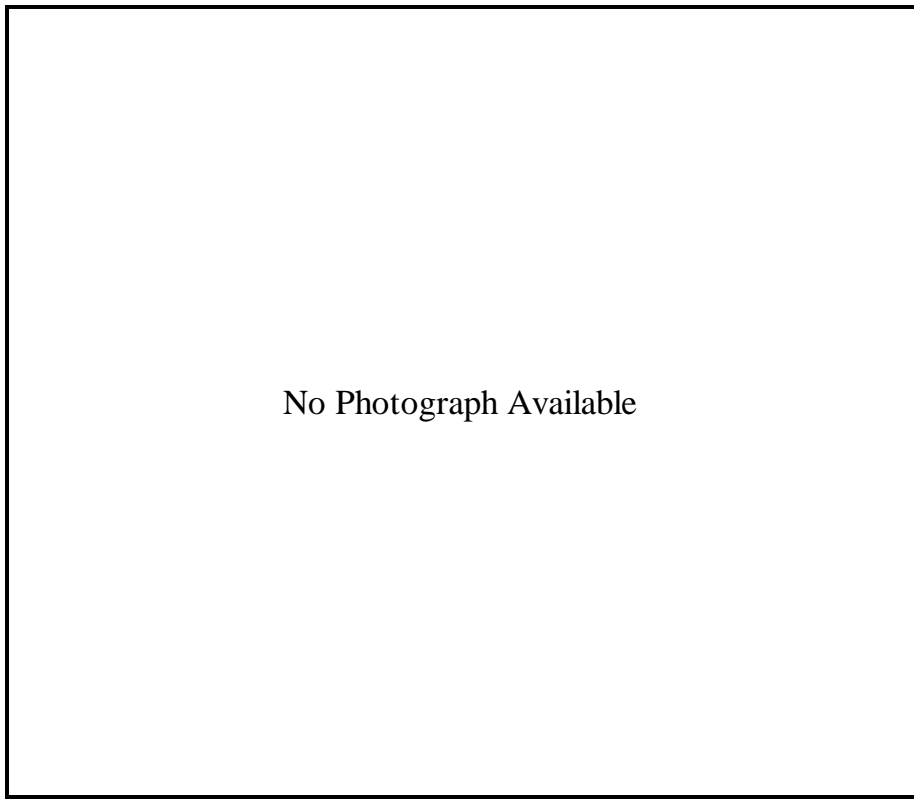

CAS Prior to Cleanup

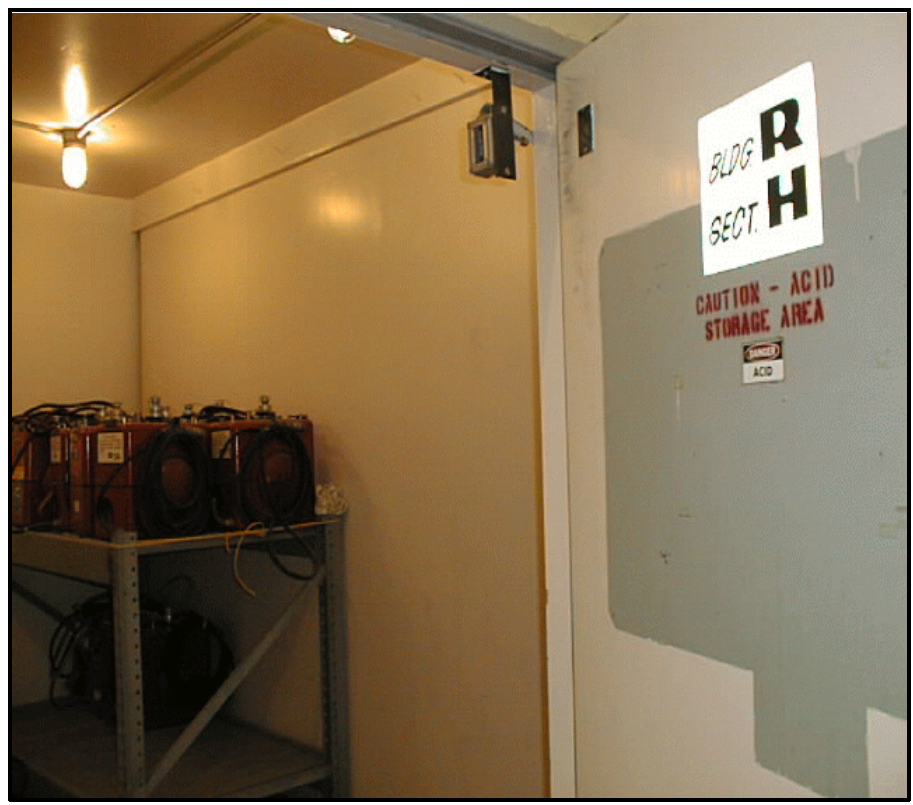

CAS After Cleanup

Photograph date: 02/05/1998

Current Site Description/Observations: The acids and bases were "issued products" for future use and were not waste. The improperly stored chemicals were removed from the storage room by J. McDow (REECo). An additional storage room was built to allow proper segregation. These compliance actions were approved by DOE/NV on 10/17/1989.

T No Further Action Required at Corrective Action Site

Brad Jackson (Industrial Sites Project Manager) 


\section{FFACO CORRECTIVE ACTION Site Housekeeping Closure Verification Form}

Closure Verification Date: 15 June 1998

CAS Number: 23-22-01

General Location: Communication Trailers, Mercury

Latitude: 36E39.807'

Longitude: -115E59.253'
CAU Number: 212

Elevation: $1,166 \mathrm{~m}$

Northing: 4057813

Easting: 590482

Coordinate/Elevation Data Obtained from NORTH AMERICAN DATUM, 1927.

Site Access Route: Turn right (east) on Tumbler Road from Mercury Highway. Proceed approximately 0.2 mile to a dirt road, turn left (north) and proceed to the large water tank on the hill. Turn right at the water tank and proceed approximately 100 yards to the communication trailer.

\begin{tabular}{|c|c|}
\hline Waste Item(s) Originally at Site & Apparent Waste Type* \\
\hline Epoxy cans and stains & Hazardous \\
\hline
\end{tabular}

* Ordinary, Scrap Metal, Asbestos, PCB, Salvageable, Hazardous, Radioactive, Mixed, Unknown, Other

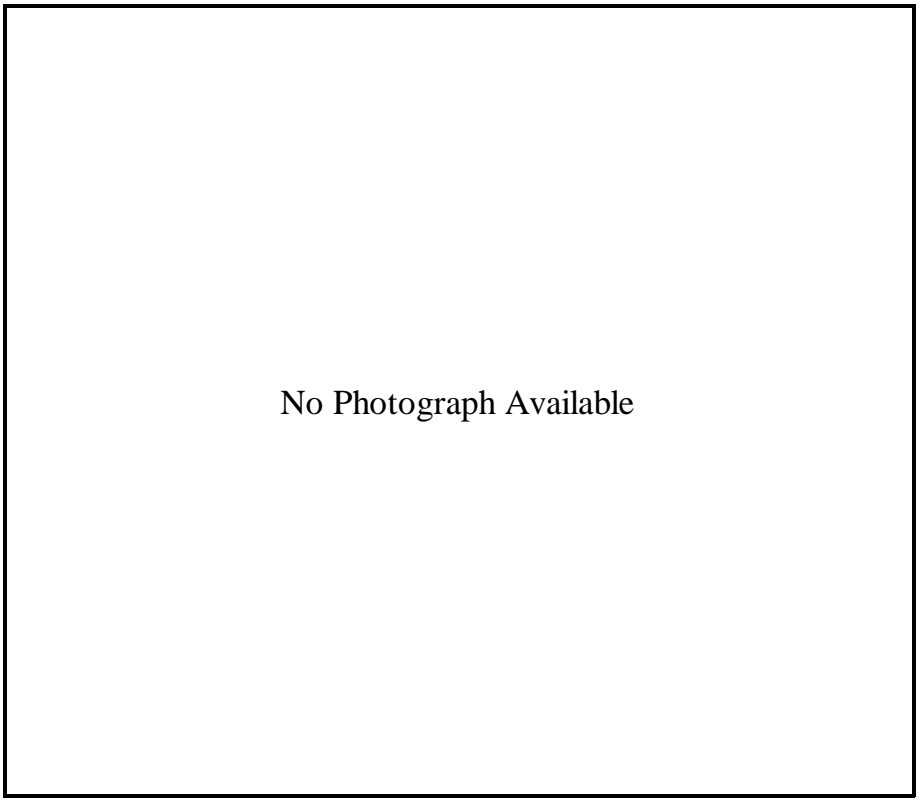

CAS Prior to Cleanup

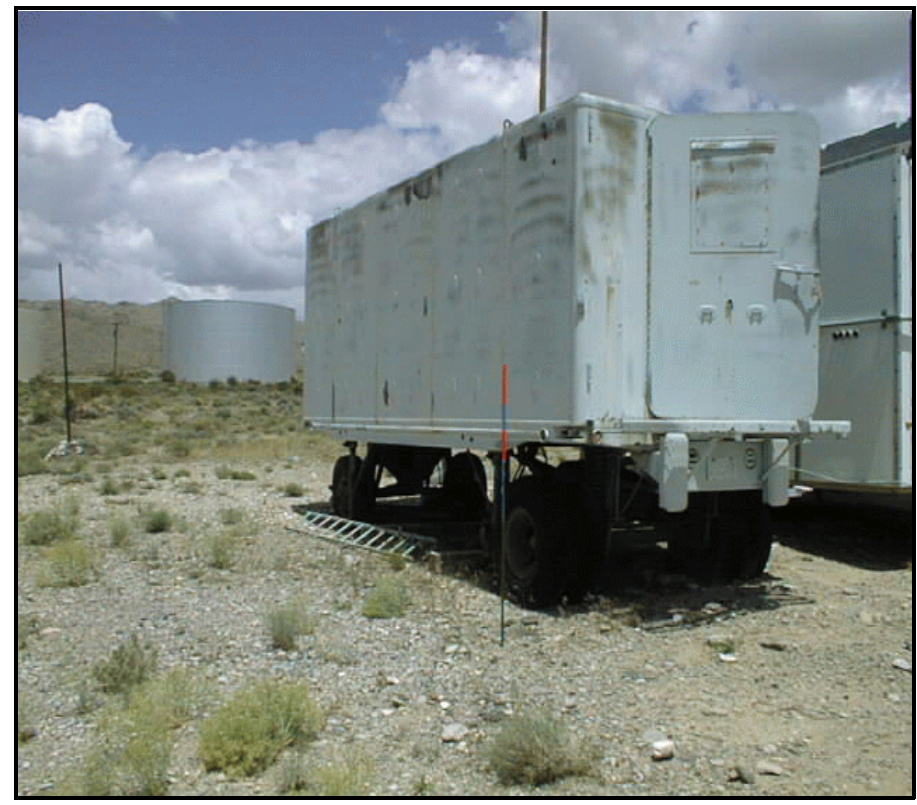

CAS After Cleanup

Photograph date: 06/11/1998

Current Site Description/Observations: The three pallets of epoxy cans were removed from the location behind the communications trailers. The small epoxy stains have been removed from the site.

\section{T No Further Action Required at Corrective Action Site}

Brad Jackson (Industrial Sites Project Manager) 


\section{Distribution}

*Distribute only if Rev. 0 is approved without changes.

\section{$\underline{\text { Copies }}$}

Paul J. Liebendorfer

2 (Controlled)

State of Nevada

Bureau of Federal Facilities

Division of Environmental Protection

333 W. Nye Lane, Room 138

Carson City, NV 89706-0851

Michael McKinnon

1 (Controlled)

State of Nevada

Bureau of Federal Facilities

Division of Environmental Protection

555 E. Washington, Suite 4300

Las Vegas, NV 89101

Sabrina Lawrence

1 (Controlled)

Environmental Restoration Division

DOE/Nevada Operations Office

P.O. Box 98518, M/S 505

Las Vegas, NV 89193-8518

Janet Appenzeller-Wing

Environmental Restoration Division

2 (Uncontrolled)

DOE/Nevada Operations Office

P.O. Box 98518, M/S 505

Las Vegas, NV 89193-8518

Brad Jackson

1 (Uncontrolled)

IT Corporation

P.O. Box 93838

Las Vegas, NV 89193

Matt Truax

1 (Uncontrolled)

IT Corporation

P.O. Box 93838

Las Vegas, NV 89193 
IT Corporation Central Files

IT Corporation

P.O. Box 93838

Las Vegas, NV 89193

Technical Information Resource Center

DOE/Nevada Operations Office

P.O. Box 98518, M/S 505

Las Vegas, NV 89193-8518

U.S. Department of Energy

Office of Scientific and Technical Information

P.O. Box 62

Oak Ridge, TN 37831

DOE Public Reading Room

P.O. Box 98521, M/S NLV040

Las Vegas, NV 89193-8521

Rosa Silver

IT Corporation

P.O. Box 93838

Las Vegas, NV 89193
1 (Uncontrolled)

1 (Uncontrolled)*

1 (Uncontrolled, electronic copy)*

1 (Controlled)*

1 (Uncontrolled)*

1 (Controlled)*

1 (Uncontrolled)* 